\title{
PDE Model Analysis Process in Image Noise Reduction
}

\author{
Lingyun Fan \\ Chongqing City Vocational College, Yongchuan, 402160, China \\ E-mail: nushui286@163.com
}

\begin{abstract}
With development of modern science and technology, partial differential equations application in digital image processing is developing rapidly in recent years. The partial differential equation of image is to deal with the fouling, the image information in the establishment of partial differential continuous mathematical model. In the solution of partial, differential equations, using specific solutions for image noise reduction, denoising, segmentation to deface the processing.
\end{abstract}

Keywords-Image Denoising; Partial Differential Equations; Image Noise; Wavelet Model; Boundary Conditions

\section{INTRODUCTION}

The image is essentially pollution damage to the image signal processing, such as noise reduction, to the damage and the like [1]. The traditional image noise using wavelet analysis and Fourier analysis method, but both methods have corresponding limitations, for stability and when the signal-frequency duality have requested [2].

The partial differential equation is a new method for image denoising, and based on the establishment of a mathematical model, the geometric characteristics of the image signal is considered. But there are some limitations on the solution of partial differential equations, Because of the huge image information [3]. It will result in infinite solutions numerical solution of partial differential equations.

In recent years, as people constantly research and the understanding of partial differential equations arises, partial differential equation is also gradually applied to the field of digital image. And its application in the field of digital image enhancing also emerges.

Among all the PDE models, the man-made structure of energy function and model established based on this function has a large proportion. Its main point is to construct an energy function, through minimize the energy functional of the structure, the researchers can achieve the purpose of realization of digital image processing such as image enhancement.

Arosio and woldl [4] put forward a kind of anisotropic diffusion model, the model use $g\left(\left|\nabla u_{\sigma}\right|\right), u_{\sigma}=G_{\sigma} \times u$ as diffusion coefficient, to reduce the noise caused by the estimated error. The improved Catte diffusion model is as follows:

$$
\begin{aligned}
& \frac{\partial u}{\partial t} \operatorname{div}\left(g\left(\left|\nabla G_{\sigma} \times u\right|\right) \nabla u\right) \\
& u(x, y, 0)=u_{0}(x, y)
\end{aligned}
$$

Which $G_{\sigma}$ is Gaussian kernel function, $G_{\sigma}=\frac{1}{4 \pi \sigma} e^{\frac{x^{2}+y^{2}}{4 \sigma}},\left|\nabla G_{\sigma} \times u\right|$, means the gradient of image with scale of $\sigma, u(x, y, 0)$ is the original image which was confirmed(Celik, T., \& Tjahjadi, T(2012)) [5].

\section{PARTIAL DIFFERENTIAL EQUATIONS}

Partial differential equation is applied to engineering modeling of Mathematical Science, but with the increase in the scope of application, it has a new development in the field of physical chemistry. Now the partial differential equation has been extended to finance, image processing, and other new areas [6]. In basic subjects, the partial differential equation is the solving process of the variation method, the ordinary differential equation, the function theory and so on [7]. It extends to other subjects, and the partial differential equation can become an independent research field.

Partial differential equation is applied to engineering modeling of Mathematical Science, but with the increase in the scope of application, it has a new development in the field of physical chemistry. Now the partial differential equation has been extended to finance, image processing, and other new areas. In basic subjects, the partial differential equation is the solving process of the variation method, the ordinary differential equation, the function theory and so on. It extends to other subjects, and the partial differential equation can become an independent research field [8].

The general form of an $n$ element containing the differential equation for the function $\varphi=\varphi\left(x_{1}, x_{2}, x_{3}, \cdots, x_{n}\right)$ is:

$F\left(x_{1}, x_{2}, x_{3}, \cdots, x_{n}, \varphi, D \varphi_{1}, D^{2} \varphi_{2}, \cdots, D^{m} \varphi_{n}\right)=0$

Among them

Second-order differential equation

$$
\sum_{i, j=1}^{n} a_{i j} \frac{\partial^{2} \varphi}{\partial x_{i} \partial x_{j}}+\sum_{i=1}^{n} b_{j} \frac{\partial \varphi}{\partial x_{i}}+c \varphi=g
$$

Among them, $a_{i j}, b_{j}$, and $c$ is the function of $x_{1}, x_{2}, x_{3}, \cdots, x_{n}$.

Fixed solution condition:

In general, the partial differential equation is a representation of the same phenomenon in a certain physical phenomenon [9]. For a partial differential equation, the solution of it often has an infinite number, but in the infinite number of solutions, the general rule of finding solutions is not very good. 
Boundary conditions:

The First Boundary Value Condition: direct physical description of system boundaries.

$$
\left.\varphi(x, t)\right|_{x \in \partial \Omega}=\psi_{1}(x, t), t \geq 0
$$

The second boundary condition: Describe the physical boundaries of the system directly reciprocal value, such as heat conduction:

$$
\left.\frac{\partial \varphi(x, t)}{\partial \vec{n}}\right|_{x \in \partial \Omega}=\psi_{1}(x, t), t \geq 0
$$

Formula wherein $\vec{n}$ is the unit normal vector $\partial \Omega$ on.

The third boundary conditions: direct physical boundaries of the system is described in combination with the physical form of reciprocal,

$$
\left.\left(\frac{\partial \varphi(x, t)}{\partial \vec{n}}+\alpha \varphi\right)\right|_{x \in \partial \Omega}=\psi_{1}(x, t), t \geq 0
$$

(1) and (2), in addition to the spatial variables to solve the partial derivatives, but also on the time of partial derivative, therefore, for some partial differential equations initial value to be set in order to solve it.

\section{BASED ON PARTIAL DIFFERENTIAL EQUATIONS TO SOLVE THE IMAGE TO DEFACE}

PDE Solve image damage is to image distortion, and then solve the partial differential equations. Its modeling process, shown in Fig. 1.

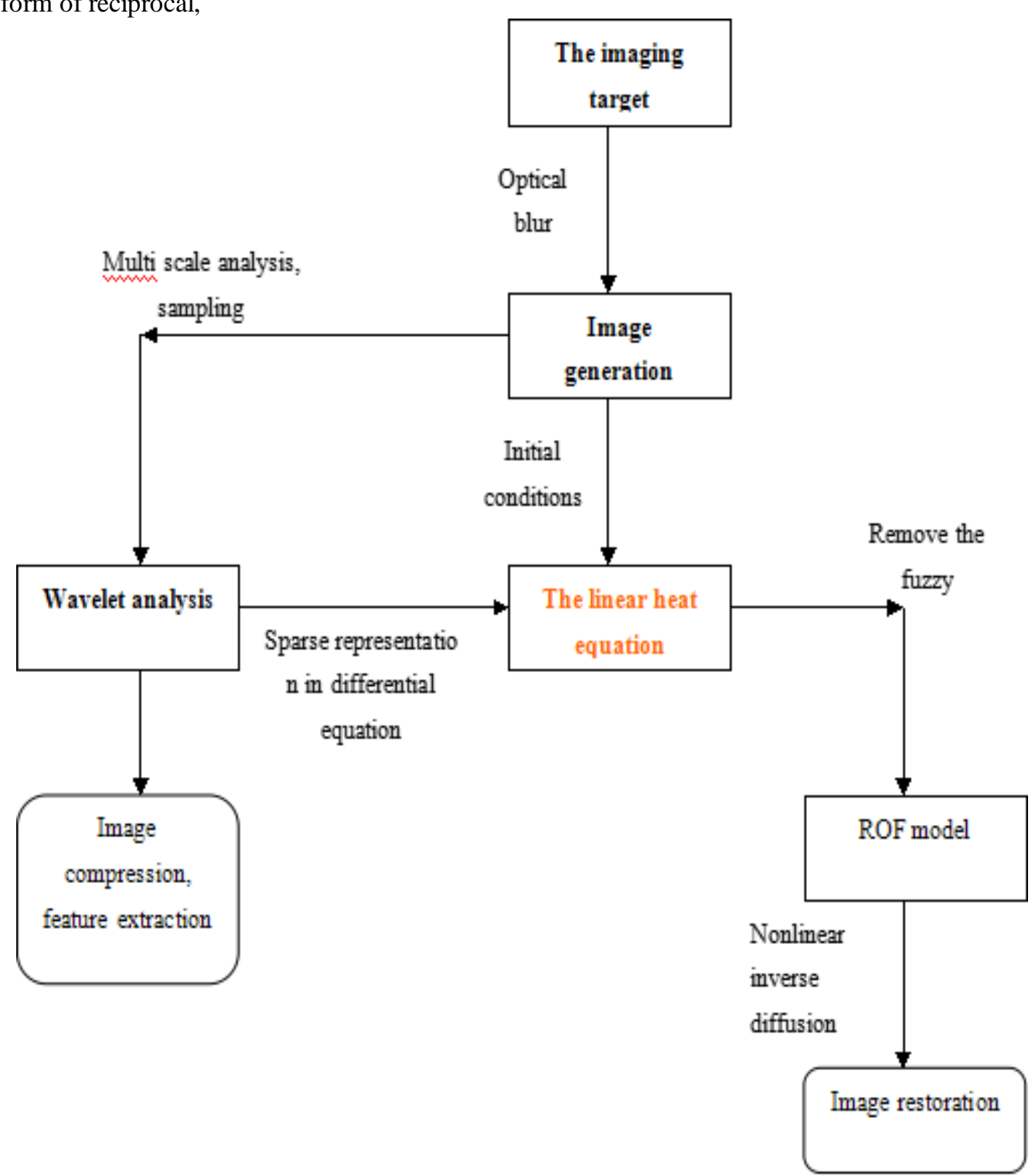

Figure 1. Picture Impairment Solving Partial Differential Equations (1) 


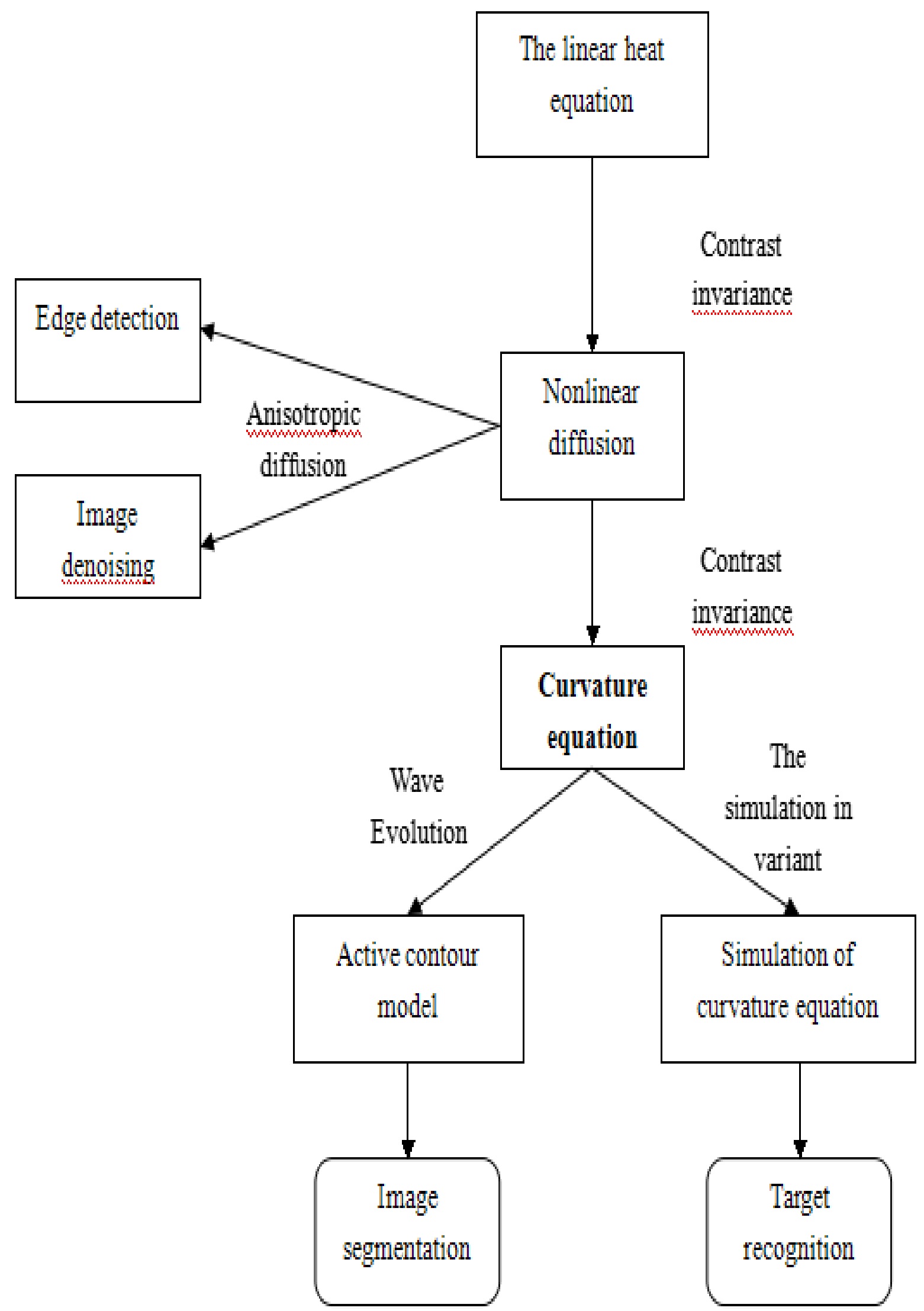

Figure 2. The Process of Solving Partial Differential Equations Damage 


\section{THE PIECEWISE LINEAR STRETCH HISTOGRAM} EQUALIZATION METHOD

The main idea of the piecewise linear stretch histogram equalization method is to divide the original image grey scale to none even $\mathrm{N}$ sections. The dividing principle is satisfy when within each segment $\Gamma_{n}$ the sum of the histogram equals to:

$$
\sum_{i \in \Gamma_{n}} h(i)=\frac{1}{N}, n=1,2, \cdots, N .
$$

The enhanced image grey scales will be evenly divided into $\mathrm{N}$ sections, then paints the starting point and end point of every grey scale sections to get the corresponding conversion function of each section, Hammond, D. K et al (2011) reported [10].

And forming an image transfer process, there will be noise, which arises inevitably, in some special conditions, high water, noise will cause great difficulties, therefore, de-noising image is an image processing the more important part of the process. It is worth noting that the image can be sharpened only if the image has high signal-to-noise ratio, or else the sharpened image will have a low signal-to-noise ratio, the reason is after sharpening the noise increased greatly compare to the signal.

\section{CONCLUSION}

For the image noise reduction method, partial differential equations is a good noise reduction algorithm, in a continuous image of the modeling process, according to setting conditions for image changes according to certain PDE, by partial differential image information equation form, to get a better picture noise. It can be said PDE for image processing, the key factor is to establish a reasonable partial differential equations, we have reached the optimal noise reduction effect.

\section{REFERENCES}

[1] Chen Qiang, Zhu Lixin, XIA deep binding Canny operator image binarization [J] Computer Aided Design and Graphics, 2005,27 (6): 1302-2306.

[2] Jian Bai, Xiang-Chu Feng. Fractional-order anisotropic diffusion for image denoising [J]. IEEE Transactions on Image Processing, 2007, 16(10):2497-2502.

[3] YANG Ying-Chun, OF SCIENTIFIC Lee of odd, etc. adaptive anisotropic diffusion image denoising model based on fractional derivative [J]. North University (Natural Science), 2011, 32 (4): 512-517.

[4] Arosio, L., Bracci, F., \& Wold, E. F. 2013. Solving the Loewner PDE in complete hyperbolic starlike domains of CN. Advances in Mathematics, 242, 209-216. DOI:10.1016/j.aim.2013.02.024

[5] Celik, T., \& Tjahjadi, T. 2012. Automatic image equalization and contrast enhancement using Gaussian mixture modeling. Image Processing, IEEE Transactions on, 21(1), 145-156. DOI: 10.1109/TIP.2011.2162419.

[6] Dong Mian, Sunyou gang, Haiyang Qiang and Xiaotian Wei. 2014. MODELING AND SIMULATION RESEARCH OF ACTIVE HEAVE COMPENSATION SYSTEM. REVIEW OF COMPUTER ENGINEER STUDIES. 1(2), 15-18.

[7] Jiao, L. C., Yang, S. Y., Liu, F., \& Hou, B. 2011. Development and prospect of compressive sensing. Dianzi Xuebao(Acta Electronica Sinica), 39(7), 1651-1662.

[8] WANG Qiao.Digital image processing [M].Beijing: SciencePress, 2009:212-231.

[9] Chen Y,,Barcelos C A Z,M air B A.Smooth ing and Edge Detection by Tim e-Varying Coup led Nonlinear D iffusion Equa-tion. Computer V ision and Im age Understanding arch ive. 2001.

[10] Hammond, D. K., Vandergheynst, P., \& Gribonval, R. 2011. Wavelets on graphs via spectral graph theory. Applied and Computational Harmonic Analysis, 30(2), 129-150 